\title{
Prekäres Wissen über prekäre Arbeit? Wissens- produktion jenseits der Arbeitssoziologie
}

\author{
Magdalena Freudenschuß
}

Prekäres Arbeiten, prekäres Leben und deshalb auch prekärer oder kein Protest? Zum politischen Handeln haben die existierenden theoretisch-analytischen $\mathrm{Zu}$ gänge zu Prekarisierung ein ambivalentes Verhältnis. In der deutschsprachigen Arbeitssoziologie wird Prekarisierung auf mehrere, sich kritisch ergänzende gesellschaftspolitische Konsequenzen hin analysiert. Marginalisierung (einzelner oder bestimmter Gruppen), Fragmentierung (der Gesellschaft), Disziplinierung (von Noch-Integrierten), Depolitisierung und Desintegration (der Prekarisierten) und Irritation (gesellschaftlicher Ordnungsmuster) sind jene sechs Momente, die am prominentesten verhandelt werden. Die Analyse, wer daraus Nutzen zieht, muss diese Liste ergänzen. Die EuroMayDays - europaweite, urbane Mobilisierungen gegen Prekarität und Prekarisierung irritieren die arbeitssoziologisch dominierende Sicht einer „Entsolidarisierung von Gesellschaft“. Prekarisierung wird in der bewegungsorientierten, theoretisch im Postoperaismus ${ }^{1}$ verankerten Debatte gezielt mit politischer Aktivität verknüpft und so auch theoretisch konzeptionalisiert.

Diese beiden Stränge der spezialisierten Wissensproduktion zu Prekarisierung - also die etablierte soziologische Forschung und die bewegungsorientierte - werden in erstaunlichem Maße voneinander isoliert geführt. Wird Prekarisierung als weitreichender Prozess diskutiert, der in Bezug auf Lebens- und nicht allein Arbeitsverhältnisse zu denken ist, kann die bewegungspolitische Wissensproduktion die arbeitssoziologische Debatte um Prekarisierung produktiv ergänzen und ausdifferenzieren. Das analytische Potenzial von Prekarisierung als Konzept zur Zeit- und Gesellschaftsdiagnose kann weiter geschärft werden, wenn - so die hier vorgelegte These - die beiden analytischtheoretischen Diskursstränge der Prekari- sierungsforschung wechselseitig rezipiert werden. Im Folgenden arbeite ich anhand der Frage nach den gesellschaftspolitischen Konsequenzen von Prekarisierung den Gewinn einer Rezeption des bewegungsorientierten Wissens zu Prekarisierung und Prekarität für den arbeitssoziologischen Diskursstrang beispielhaft heraus.

\section{ENTSOLIDARISIERUNG DURCH PREKARISIERUNG}

Marginalisierung und Disziplinierung verweisen beide darauf, dass nicht allein der prekär beschäftigte Teil der Bevölkerung von Prekarisierung betroffen ist. Prekarisierung wird in der Arbeitssoziologie häufig als neue soziale Frage (Castel) gefasst, weil sie für die einen soziale und ökonomische Marginalisierung, für die Noch-Integrierten Disziplinierung bedeutet. Über die Momente von Marginalisierung und Disziplinierung hinaus wird in der deutschsprachigen Arbeitssoziologie eine Fragmentierung von Gesellschaft konstatiert, insofern, als die Interessen prekarisierter Subjekte sehr unterschiedlich angelegt sind. Von hochqualifizierten Projektarbeiterinnen und Projektarbeitern oder Praktikantinnen und Praktikanten über Leiharbeiterinnen und Leiharbeiter bis hin zu Erwerbsarbeitslosen reicht die Spannbreite prekärer oder prekarisierter Positionen. Sie verfügen zudem über unterschiedliche Ressourcen zum Umgang mit ihrer unsicheren Beschäftigungs- und Lebenssituation.

Die Fragmentierung prekarisierter Subjekte wird als einer der zentralen Gründe für eine Depolitisierung beziehungsweise für eine Reduktion ihrer Handlungsfähigkeit angeführt. Folgen wir dieser Argumentation, so treffen wir auf die Figur eines prekären Subjekts, dessen soziale Teilhabe systematisch untergraben wird. Seine politische Handlungsfähigkeit gilt als eingeschränkt, denn kollektive Auflehnung setze „ein Mindestmaß an Hoffnung und Glauben an die Zukunft ${ }^{\text {“2 }}$ voraus, das unter prekären Lebensbedingungen von Betroffenen nicht mehr aufgebracht werden könne. Die materielle Unsicherheit, die aus der Erwerbsarbeitssituation der Einzelnen resultiert, sowie umfassende Individualisierungsprozesse trügen zu Entsolidarisierung und Entpolitisierung bei.

Kritik an diesen Interpretationen der Prekarisierung kommt aus der feministischen Arbeitsforschung. Ein akteursorientierter Ansatz erlaubt eine Sicht auf Prekarisierung als Prozess, der Lebensverhältnisse vervielfältigt und mitunter Irritationen und Verschiebungen innerhalb tradierter Geschlechterordnungen ermöglicht. ${ }^{3}$ Komplexe gesellschaftliche Dynamiken, nicht allein repressive, passivierende Konsequenzen ergeben sich aus dem Wandel von Arbeit. Auch Emanzipationsgewinne, neue Widerstandsstrategien und Handlungsräume eröffnen sich für prekarisierte Subjekte: Mehr Eigenständigkeit durch Erwerbsarbeit, neue Allianzen zwischen Erwerbslosen und Kreativen,

1 Häufig zitierte Autoren des Postoperaismus (auch "autonomous marxism “) sind Antonio Negri und Michael Hardt, Paolo Virno, Maurizio Lazzarato. 2 Bourdieu, P. (2004): Gegenfeuer, Konstanz, S. 108.

3 Vgl. Völker, S. (2009): „Entsicherte Verhältnisse“. Impulse des Prekarisierungsdiskurses für eine geschlechtersoziologische Zeitdiagnose, in: Aulenbacher, B./Wetterer, A. (Hrsg.): Arbeit. Perspektiven und Diagnosen der Geschlechterforschung, Münster, S. 268-286.

\footnotetext{
Magdalena Freudenschuß, Politikwissenschaftlerin, promoviert an der HumboldtUniversität zu Berlin, Mitherausgeberin der Femina Politica. Arbeitsschwerpunkte: Prekarisierung, feministische Theorie, Diskursforschung. e-mail: m_freudenschuss@web.de
} 
neue Strategien des Sich-Entziehens oder Protestierens lassen sich beobachten.

In diesen arbeitssoziologischen Konzeptualisierungen wird Prekarisierung als Gesellschaft strukturierender Prozess theoretisiert, der in der Regel nationalstaatliche Gesellschaften als Gesamte, aber verschiedene soziale Gruppen auch auf sehr unterschiedliche Weise betrifft. Kollektives politisches Handeln scheint allerdings nicht bruchlos möglich.

\section{POLITISIERUNG ANGESICHTS VON PREKARISIERUNG}

Dennoch: Im Rahmen der EuroMayDays gehen sich als prekarisiert verstehende Menschen seit 2001 am 1. Mai auf die Straße. Sie treten als „die Prekären“ auf, demonstrieren gegen ihre prekäre Arbeitsund Lebenssituationen, fordern neue soziale Rechte und beziehen sich explizit auf ein kollektives Subjekt. In den Folgejahren vernetzten sich europaweit in einer wachsenden Zahl von Städten Aktivistinnen und Aktivisten mit ähnlichen Anliegen. Allen Aktivitäten gemeinsam - ob Demonstrationen, Wissensproduktionen oder anderen Formen von Aktion und Mobilisierung - ist der Bezug auf Prekarisierung: Die entsprechenden Begrifflichkeiten sind nicht nur Teil der politischen Mobilisierung zu den EuroMayDay-Demonstrationen, sondern werden auch theoretisch ausgearbeitet. Neben den theoretisch-philosophischen Arbeiten des Postoperaismus stellen Mobilisierungstexte (zumindest partiell) Analysen des sozialen Wandels zur Verfügung. Geschichte und Selbstdokumentation ${ }^{4}$ der Bewegung machen deutlich, dass diese sich mit Bezug auf diese Begrifflichkeiten erst konstituiert. Sie stellen eine Art roten Faden zwischen verschiedenen Orten der Mobilisierung dar, sie erlauben eine Verkettung von Problemlagen und Kämpfen. Die EuroMayDay-Bewegung arbeitet mit einem verkettenden Subjektbegriff. Er adressiert verschiedene soziale Ungleichheiten in ihren Verschränkungen. Indem diese parallel thematisiert, nicht aber hierarchisiert werden, können Singularitäten verkettet, in einer neuen Form der Organisierung miteinander verbunden werden. Eine politische Praxis jenseits von klassischer Identitätspolitik entsteht.

In diesem Diskursstrang zu Prekarisierung gilt das prekarisierte Subjekt als aktives, politisch mobilisierbares. Die Bewegung entwickelt selbst „Subjektivie- rungsangebote und diskursive Raster [...], die es ermöglichen, „die Prekären“ als soziale Akteure zu denken“. ${ }^{5}$ Dem entspricht ein Sprechen als „wir“, das einschließend, gleichzeitig differenzierend sein will:

"We are those precarious people. We are the women of Europe in a feminized workforce and economy that nevertheless reserves to $\mathrm{xx}$ people more discriminatory pay and roles than to domineering $x y$ people. We are the consumerized younger generation left out of the political and social design of a gerontocratic and technocratic Europe. We are the first-generation Europeans coming from five continents and, most crucially, the seven seas. We are the middle-aged being laid off from once secure jobs in industry and services. We are the people that don't have (and mostly don't want) long-term jobs, and so are deprived of basic social rights such as maternity or sick leave or the luxury of paid holidays. We are hirable on demand, available on call, exploitable at will, and fireable at whim. We are the precariat. “6

In diesem Zitat werden Selbstverständnis und Anspruch vieler Aktivistinnen und Aktivisten deutlich: Die geteilte Position des Prekär-Seins wird in der Differenzierung und gleichzeitig in der solidarischen Identifikation eines „we are“ zur Identität. Geschlecht, Herkunft, Alter erlauben es, spezifische Subjektpositionen innerhalb der Gruppe von prekären Subjekten zu bestimmen. Differenzen werden sichtbar gemacht und mit Blick auf die Arbeits- und Lebensbedingungen in einer gemeinsamen Position miteinander verkettet. Charakteristisch für die EuroMayDays ist der Aufruf, eine neue Form kollektiven Handelns zu erfinden, die sich nicht auf enge identitäre Konzepte („Frauen“ oder „Arbeiter“) stützt.

Prekäre Subjektpositionen werden weit gefasst, beispielhaft konkretisiert, abstrakter wieder erweitert: So ist im Aufruf zum Amsterdamer MayDay 2005 zu lesen, dass sowohl bezahlte wie unbezahlte Arbeit unter prekären Bedingungen organisiert ist. Dieser Verweis hebt klassische Grenzziehungen auf. Potenziell steht nicht allein Erwerbsarbeit auf der Agenda der Protestierenden, sondern das aktuelle Arrangement von Arbeit insgesamt. Damit ist klar, dass nicht allein die Position im Verhältnis von Kapital und Arbeit die soziale Position und das politische Interesse der Prekären bestimmt: „Pflege, Wohnung, Migration, Einkommen, Geschlecht, Mobilität, prekäre Beschäftigung und Sexualität verflechten sich zu einer möglichen Karte von Konflikten, Herausforderungen und der Eroberung neuer sozialer Rechte “ .7 Die Thematisierung von Prekarisierung erfolgt als komplexe Kartographie von mehreren Faktoren, die zu unterschiedlichen Konstellationen führen.

\section{ENTSOLIDARISIERUNG UND POLITISIERUNG}

Prekarisierung kann ausgehend von dieser Debatte zusätzlich als politisierendes Phänomen gelesen werden. Der vielschichtige soziale Wandel impliziert Protest damit ebenso, wie er Arbeit und Leben tangiert. Marginalisierung und Privilegierung, Fragmentierung, Disziplinierung und befreiende Irritation, Desintegration, Depolitisierung wie Politisierung sind Momente dieses Wandels sozialer Verhältnisse. Prekarisierung kann in diesem Sinne als Zeitdiagnose funktionieren, die mitunter uneindeutige gesellschaftspolitische Entwicklungen und deren Auswirkungen auf individuelle Lebenslagen und Möglichkeiten konzeptionell bündelt. Prekarisierung im Lebenszusammenhang als uneindeutig, ungleichzeitig und komplex zu denken, wurde bereits von der feministischen Arbeitsforschung systematisch gefordert. Die Rezeption bewegungspolitischer, postoperaistischer Debatten, die bislang eine rare Ausnahme ${ }^{8}$ in der deutschsprachigen Arbeitssoziologie darstellt, erweitert und schärft die Untersuchung von Prekarisierung im Sinne einer soziologischen Zeitdiagnose.

4 Luzerner Forschungsprojekt „Protest as medium - media as protest “, Online-Archiv der EuroMayDays, Verweise auf Bewegungstexte sind abrufbar unter: http://unilu.strg.ch/.

5 Adolphs, St.; Hamm, M. (2008): Prekäre Superhelden. Zur Entwicklung politischer Handlungsmöglichkeiten in postfordistischen Verhältnissen, in: Altenhain, C./Danilina, A./Hildebrandt, E./ Kautsch, S./Müller, A./Roschner, T. (Hrsg.): Von "Neuer Unterschicht" und Prekariat. Gesellschaftliche Verhältnisse und Kategorien im Umbruch. Kritische Perspektiven auf aktuelle Debatten, Bielefeld, S. 175

6 Foti, A. (2005): MAYDAY MAYDAY: euro flex workers, time to get a move on! (04), http:// republicart.net/disc/precariat/foti01_en.pdf.

7 O.A. (2008): Euromayday Terrassa 08-L@s precari@s se van de marcha (Call Terrassa, 1.5.2008), Euromayday Online Archive, http:// unilu.strg.ch/. Übersetzung der Autorin.

8 Vgl. Altenhain, C. et al. (Hrsg.) (2008): Von „Neuer Unterschicht", a.a.O.; Manske, A./ Pühl, K. (Hrsg.) (2010): Prekarisierung zwischen Anomie und Normalisierung, Münster. 